\title{
ANDERSON INEQUALITY IS STRICT FOR GAUSSIAN AND STABLE MEASURES
}

\author{
MACIEJ LEWANDOWSKI, MICHAL RYZNAR, AND TOMASZ ŻAK
}

(Communicated by Lawrence Gray)

\begin{abstract}
Let $\mu$ be a symmetric Gaussian measure on a separable Banach space $(E,\|\cdot\|)$. Denote $U=\{x:\|x\|<1\}$. Then for every $x \in \operatorname{supp} \mu$, $x \neq 0$, the function $t \rightarrow \mu(U+t x)$ is strictly decreasing for $t \in(0, \infty)$. The same property holds for symmetric p-stable measures on $E$. Using this property we answer a question of $\mathrm{W}$. Linde : if $\int_{U+z} x d \mu(x)=0$, then $z=0$.
\end{abstract}

\section{NOTATION AND BASIC PROPERTIES OF GAUSSIAN MEASURES}

We start by recalling some basic notation and facts concerning Gaussian measures on Banach spaces. For the proofs the reader may consult [LePage] or [Bor] .

Throughout the paper $(E,\|\cdot\|)$ denotes a separable Banach space. Let $\mu$ be a symmetric Gaussian measure on $E$. By supp $\mu$ we denote the support of $\mu$ which is a linear subspace of $E$. Let us mention that $x \in \operatorname{supp} \mu$ if and only if $\mu\{y \in E:\|y-x\|<\varepsilon\}>0$ for every $\varepsilon>0$. Let $E_{2}^{*}(\mu)$ denote a closure of $E^{*}$ (topological dual of $E$ ) in $L_{2}(\mu)$. For every $f \in E_{2}^{*}(\mu)$ we define an operator $Q$ by the formula $Q f=\int_{E} f(x) x d \mu(x)$. Then $Q$ maps $E_{2}^{*}(\mu)$ onto a subspace of $E$ which we denote $H_{\mu}$. Let $Q^{\prime}: E^{*} \rightarrow E_{2}^{*}(\mu)$ be the natural injection. Then the covariance operator $R$ of $\mu$ is defined by the following formula: $R=Q Q^{\prime}: E^{*} \rightarrow H_{\mu}$. For every $h \in H_{\mu}$ there exists the unique element $\tilde{h} \in E_{2}^{*}(\mu)$ such that $Q \tilde{h}=h$. Then the formula $\left\langle h_{1}, h_{2}\right\rangle=\int_{E} \tilde{h}_{1}(x) \tilde{h}_{2}(x) d \mu(x)$ defines a scalar product in $H_{\mu}$ and this, in turn,

defines a norm on $H_{\mu}:\|h\|_{\mu}=\langle h, h\rangle^{\frac{1}{2}}$. $H_{\mu}$ equipped with this norm is a Hilbert space, we call it the reproducing kernel Hilbert space (RKHS) of $\mu$. $H_{\mu}$ is dense in the support of $\mu$ and consists of the admissible translates of the measure $\mu$. For $y \in E$ let us denote by $\mu_{y}$ the translated measure defined by $\mu_{y}(\cdot)=\mu(\cdot+y)$. There holds the following important fact.

Proposition 1 (Cameron-Martin formula [C-M]). For each $h \in H_{\mu}$ the measure $\mu_{h}$ is absolutely continuous with respect to $\mu$ and for every measurable set $A$ we

Received by the editors February 26, 1992 and, in revised form, January 10, 1994.

1991 Mathematics Subject Classification. Primary 60B11.

Key words and phrases. Gaussian measures, stable measures, Anderson inequality.

This research is supported by a KBN Grant. 
have

$$
\mu_{h}(A)=\exp \left(-\frac{1}{2}\|h\|_{\mu}^{2}\right) \int_{A} e^{-\tilde{h}(x)} d \mu(x) .
$$

Applying the above proposition we obtain the following corollary.

Corollary 1. Let $f \in E^{*}$ with $\int_{E} f^{2}(x) d \mu(x)=1$. Set $z_{0}=R f \in H_{\mu}$ and $\gamma=\mathscr{L}\left(g z_{0}+X\right)$, where $\mathscr{L}(X)=\mu$ and $g$ is the standard Gaussian random variable on the real line, independent of $X$. Then $\gamma$ is absolutely continuous with respect to $\mu$ and

$$
\frac{d \gamma}{d \mu}(y)=\frac{1}{\sqrt{2}} \exp \left(\frac{f^{2}(y)}{4}\right) .
$$

The following fact may be derived from the so-called log-concavity property of Gaussian measures (see e.g. [Bor], Theorem 3.2), but it was proved for the first time by Anderson [And] for unimodal distributions on $R^{n}$, hence we call it the Anderson property of Gaussian measures.

Proposition 2 (Anderson property of Gaussian measures). If $\mu$ is a symmetric Gaussian measure on $E$ and $C$ is a convex, symmetric measurable subset of $E$, then for every $x \in E$

$$
\mu(C) \geq \mu(C+x)
$$

\section{Main Result}

Our main result states that the above inequality must be strict if only $x \neq 0$. We precede the proof of this fact with a lemma. Let us denote $U_{t}=\{x \in E$ : $\|x\|<t\}$ and $U_{1}=U$.

Lemma 1. Suppose that $x_{0} \neq 0$ is such that $\left(\left(U+x_{0}\right) \backslash U\right) \cap \operatorname{supp} \mu \neq \varnothing$. Then there exists a functional $f \in E^{*}$ such that for some $u_{0}>0$ :

$$
U \subset\left\{|f|<u_{0}\right\}, \quad \mu\left(\left(U+x_{0}\right) \cap\left\{|f|<u_{0}\right\}\right)>0 \text { and } \int_{E} f^{2}(x) d \mu(x)=1 .
$$

Proof. Let $y \in\left(\left(U+x_{0}\right) \backslash \bar{U}\right) \cap \operatorname{supp} \mu$. Since $\left(U+x_{0}\right) \backslash \bar{U}$ is open, there exists a closed ball $\bar{U}_{\{y\}}$ with center at $y$ such that $\bar{U}_{\{y\}} \subset\left(\left(U+x_{0}\right) \backslash \bar{U}\right)$. Now the conclusion of the lemma yields from the convexity of $\bar{U}$ and $\bar{U}_{\{y\}}$, the Hahn-Banach theorem and the fact that $\mu\left(\bar{U}_{\{y\}}\right)>0$.

Now we are able to state the main result of this paper.

Theorem 1. Let $x_{0} \in E$. Then $\mu\left(U+x_{0}\right)=\mu(U)$ if and only if

$$
\left.\mu\left(\left(U+x_{0}\right) \backslash U\right)\right)+\mu\left(U \backslash\left(U+x_{0}\right)\right)=0 .
$$

Before proving Theorem 1 we derive an important corollary.

Corollary 2. Let $x_{0} \neq 0$. Then $\mu\left(U+x_{0}\right)<\mu(U)$ in the following cases:

(1) $x_{0} \in \operatorname{supp} \mu$.

(2) $\|\cdot\|$ is strictly convex. 
Proof of the corollary. (1) It is clear that for some $\lambda>1$ we have

$$
\left\|\lambda x_{0}-x_{0}\right\|=(\lambda-1)\left\|x_{0}\right\|<1 \text { and } \lambda\left\|x_{0}\right\|>1 \text {. }
$$

Therefore $\lambda x_{0} \in\left(U+x_{0}\right) \backslash \bar{U}$. Since supp $\mu$ is a linear subspace of $E$, we get $\mu\left(\left(U+x_{0}\right) \backslash U\right)>0$.

(2) There exists an $y \in \partial U \cap \operatorname{supp} \mu$. (If it were not true, then $\mu$ would be $\delta_{0}$.) If $y \notin \partial\left(U+x_{0}\right)$, then either $\lambda y \in\left(U+x_{0}\right) \backslash \bar{U}$ for some $\lambda>1$ or $\lambda y \in U \backslash\left(\overline{U+x_{0}}\right)$ for some $\lambda<1$. In both cases $\lambda y \in \operatorname{supp} \mu \cap$ $\left.\left[\left(U \backslash\left(\overline{U+x_{0}}\right)\right) \cup\left(U+x_{0}\right) \backslash \bar{U}\right)\right]$ and the last set has positive measure. Now we assume that $y \in \partial U \cap \partial\left(U+x_{0}\right)$. From the strict convexity of the norm $\left\|y+x_{0}\right\|+\left\|y-x_{0}\right\|>2\|y\|=2$ and then $\left\|x_{0}+y\right\|>1$. Clearly, for some $\lambda$, $0<\lambda<1$, we have $\left\|-\lambda y-x_{0}\right\|>1$ and $\|-\lambda y\|=\lambda<1$. This means that $-\lambda y \in\left(U \backslash \overline{\left(U+x_{0}\right)}\right) \cap \operatorname{supp} \mu$. Hence $\mu\left(U \backslash\left(U+x_{0}\right)\right)>0$.

Proof of Theorem 1. Suppose that $\mu\left(\left(U+x_{0}\right) \backslash U\right)>0$. Then $\left(\left(U+x_{0}\right) \backslash \bar{U}\right) \cap$ supp $\mu \neq \varnothing$ and, by Lemma 1 , we find an $f \in E^{*}$ such that $\int_{E} f^{2}(x) d \mu(x)=1$ and for some $u_{0}>0$

$$
U \subset\left\{|f| \leq u_{0}\right\} \text { and } \mu\left(\left(U+x_{0}\right) \cap\left\{|f|>u_{0}\right\}\right)>0 .
$$

Let $\gamma$ be a symmetric Gaussian measure defined in Corollary 1 , and let $h(y)=$ $\frac{d y}{d \mu}(y)=\frac{1}{\sqrt{2}} \exp \left(\frac{f^{2}(y)}{4}\right)$. Denote $D_{t}=\{y \in E: h(y) \leq t\}, t>0$. From the form of $h$ it is clear that $D_{t} \subset D_{t^{\prime}}$ for $t \leq t^{\prime} ; D t$ are symmetric, convex sets (strips in $E$ ) and there exists some $T_{0}>0$ such that

$$
\left(U+x_{0}\right) \subset D_{T_{0}} \text { and } U \subset D_{T_{0}} .
$$

Let us consider the following distribution functions:

$$
F_{x_{0}}(t)=\mu\left(\left(U+x_{0}\right) \cap D_{t}\right) \text { and } F(t)=\mu\left(U \cap D_{t}\right), \quad t>0 .
$$

From the convexity of $D_{t}$ and $U$ it follows that

$$
\frac{1}{2}\left(\left(U+x_{0}\right) \cap D_{t}\right)+\frac{1}{2}\left(\left(U-x_{0}\right) \cap D_{t}\right) \subset U \cap D_{t},
$$

and then we can apply the log-concavity property ([Bor], Theorem 3.2 ) to conclude that

$$
\mu\left(U \cap D_{t}\right) \geq \mu^{\frac{1}{2}}\left(\left(U+x_{0}\right) \cap D_{t}\right) \mu^{\frac{1}{2}}\left(\left(U-x_{0}\right) \cap D_{t}\right) .
$$

Next, by the symmetry of $U$ and $D_{t}$, the last statement is equivalent to

$$
F_{x_{0}}(t) \leq F(t) \text {. }
$$

Using Proposition 2 and Corollary 1 and integrating by parts we get

$$
\begin{aligned}
0 \geq \gamma\left(U+x_{0}\right)-\gamma(U) & =\int_{U+x_{0}} h(y) d \mu(y)-\int_{U} h(y) d \mu(y) \\
& =\int_{0}^{T_{0}} t d F_{x_{0}}(t)-\int_{0}^{T_{0}} t d F(t) \\
& =T_{0}\left[F_{x_{0}}\left(T_{0}\right)-F\left(T_{0}\right)\right]-\int_{0}^{T_{0}}\left(F_{x_{0}}(t)-F(t)\right) d t .
\end{aligned}
$$


Knowing that $F_{x_{0}}\left(T_{0}\right)=\mu\left(U+x_{0}\right)$ and $F\left(T_{0}\right)=\mu(U)$ we can rewrite the last inequality as

$$
\mu(U)-\mu\left(U+x_{0}\right) \geq \frac{1}{T_{0}} \int_{0}^{T_{0}}\left(F(t)-F_{x_{0}}(t)\right) d t .
$$

From (1) and (3) it is clear that the integral must be strictly positive. To complete the proof we have to consider the situation when $\mu\left(U \backslash\left(U+x_{0}\right)\right)>0$ and $\mu\left(\left(U+x_{0}\right) \backslash U\right)=0$. But then

$$
\mu(U)-\mu\left(U+x_{0}\right)=\mu(U)-\mu\left(U \backslash\left(U+x_{0}\right)\right)>0 .
$$

The proof is complete.

The same property holds for measures which are mixtures of Gaussian ones. For example we have the following.

Corollary 3. Let $\mu$ be a symmetric p-stable measure on $E$. Then for every $x \in \operatorname{supp} \mu$ we have

$$
\mu(U)>\mu(U+x) .
$$

Proof. By the well-known representation of symmetric stable measures as a mixture of Gaussian (compare e.g. [LP-W-Z] or [Szt] ) we have for measurable set $A: \mu(A)=\int_{T} \gamma_{t}(A) d m(t)$, where $\gamma_{t}$ are symmetric Gaussian, $m$ is a finite measure on some measurable space $T$, and $\operatorname{supp} \gamma_{t}=\operatorname{supp} \mu$ for $m$-almost all $t$. Because $\gamma_{t}(U)-\gamma_{t}(U+x)>0$, it follows that $\mu(U)-\mu(U+x)>0$ as we claimed.

Remark. In order to get the strict Anderson inequality we must assume that the translate $x$ or the norm $\|\cdot\|$ have some additional properties.

Example. Let $E=R^{2}$ be equipped with the maximum norm $\|(x, y)\|=$ $\max (|x|,|y|)$. Let $\mu$ be the one-dimensional standard Gaussian measure that is regarded as a measure on $R^{2}$ and has the axis $O x$ as its support. Then for every $t, \quad 0<t<1, \mu\left(U_{1}+(0, t)\right)=\mu\left(U_{1}\right)$, because $U_{1} \cap \operatorname{supp} \mu=$ $\left[U_{1}+(0, t)\right] \cap \operatorname{supp} \mu$. Observe that in this example neither the norm is strictly convex nor $(0, t)$ belongs to the $\operatorname{supp} \mu$. However, when $z$ has a non-zero second coordinate, then $\mu(U+z)<\mu(U)$.

\section{SOLUTION OF A PROBLEM OF LINDE}

In his paper [Lin] Linde examined the smoothness properties of the function $x \rightarrow \mu\left(\bar{U}_{s}+x\right)$ for $\mu$ Gaussian. Namely, he showed that this function is Gateaux differentiable at every $x \in \operatorname{supp} \mu$, that is, there exists a continuous linear functional $d(s, x)(\cdot)$ such that

$$
d(s, x)(y)=\lim _{\varepsilon \rightarrow 0} \frac{1}{\varepsilon}\left[\mu\left(\bar{U}_{s}+x+\varepsilon y\right)-\mu\left(\bar{U}_{s}+x\right)\right] .
$$

By virtue of the log-concavity, in the separable Banach spaces the Gaussian measure of $\partial\left(U_{s}+x\right)$ is zero for every $x \in \operatorname{supp} \mu$ and $s>0$ (compare [HJS-D] for the proof of this fact if $x=0$ ), hence Linde's result is also valid for $U_{s}$ instead of $\bar{U}_{s}$. Linde showed that the differential $d(s, x)(\cdot)$ is non-trivial 
if $\|x\|>s$ and asked about the case $\|x\| \leq s$. Now we show that $d(s, x)$ is a non-zero functional if only $x \neq 0$, what is more, we show that $d(s, x)(x)<0$.

The next theorem answers in positive the question of Linde we mentioned earlier.

Theorem 2. In the above setting, for every $x \in \operatorname{supp} \mu, x \neq 0$,

$$
d(s, x)(x)<0 \text {. }
$$

Proof. Consider a function $f_{x}(t)=\frac{1}{\mu\left(U_{s}+t x\right)}$ for $x \neq 0$. Then $f_{x}$ is even, convex and strictly increasing on $(0, \infty)$. It is clear that $f_{x}$ is even and tends to infinity as $t \rightarrow \infty$. By log-concavity of the measure $\mu$ we conclude that the function $t \rightarrow \log \mu\left(U_{s}+t x\right)$ is concave, hence $\frac{1}{\mu\left(U_{s}+t x\right)}=\exp \left(-\log \mu\left(U_{s}+t x\right)\right)$ is convex. But Theorem 1 implies that $f_{x}(t)>f_{x}(0)$ for every $t>0$, hence $f_{x}$ is strictly increasing (because it is convex).

Next, by easy computations we get for $x \in \operatorname{supp} \mu$ :

$$
d(s, x)(x)=\lim _{\varepsilon \rightarrow 0} \frac{1}{\varepsilon}\left[\mu\left(U_{s}+x+\varepsilon x\right)-\mu\left(U_{s}+x\right)\right]=\left(f_{x}(t)^{-1}\right)_{t=1}^{\prime} .
$$

But $f_{x}$ is strictly increasing and convex, hence $\left(f_{x}\right)_{t=1}^{\prime}>0$, which implies that

$$
d(s, x)(x)=-\frac{f_{x}^{\prime}(1)}{f_{x}^{2}(1)}<0 .
$$

As it was shown in Linde's paper [Lin] it is easy to compute $d(s, z)(h)$ for $h \in H(\mu)$. Namely, using the Cameron-Martin formula (Proposition 1) we get

$$
d(s, z)(h)=-\int_{U_{s}+z} \tilde{h}(x) d \mu(x)=-\tilde{h}\left(\int_{U_{s}+z} x d \mu(x)\right) .
$$

Now we show that for Gaussian measure $\mu$ the condition $\int_{U_{s}+z} x d \mu(x)=0$ implies $z=0$.

Theorem 3. Let $\mu$ be a symmetric Gaussian measure on $E$. If $z \in \operatorname{supp} \mu$, then $\int_{U_{s}+z} x d \mu(x)=0$ implies $z=0$.

Proof. Arguing as at the beginning of the proof of Theorem 2 we infer that the function $g_{h}(t)=\frac{1}{\mu\left(U_{s}+z+t h\right)}$ is convex for all $h \in H_{\mu}$. If $z \neq 0$, then, by Theorem 1, $\mu\left(U_{s}+z\right)<\mu\left(U_{s}\right)$, hence for at least one $h_{0} \in H_{\mu}$, the derivative $g_{h_{0}}^{\prime}(0)$ is not equal to zero ( $g_{h_{0}}$ attains its minimum at some $t \neq 0$ ), so that $0 \neq d(s, z)\left(h_{0}\right)=-\tilde{h}_{0}\left(\int_{U_{s}+z} x d \mu(x)\right)$ which, of course, is equivalent to the condition $\int_{U_{s}+z} x d \mu(x) \neq 0$.

\section{REFERENCES}

[And] T. W. Anderson, The integral of a symmetric unimodal function over a symmetric convex set and some probability inequalities, Proc. Amer. Math. Soc. 6 (1955), 170-176.

[Bor] C. Borell, Gaussian Radon measures on locally convex spaces, Math. Scand. 38 (1976), 265-284.

[C-M] R. H. Cameron and W. T. Martin, The orthogonal development of non-linear functionals in series of Fourier-Hermite functionals, Ann. of Math. 48 (1947), 385-392. 
[HJ-S-D] J. Hoffmann-Jørgensen, L. A. Shepp, and R. M. Dudley, On the lower tail of Gaussian seminorms, Ann. Probab. 7 (1979), 319-342.

[LePage] R. Le Page, Note relating Bochner integrals and reproducing kernels to series expansions on a Gaussian Banach space, Proc. Amer. Math. Soc. 32 (1972), 285-288.

[LP-W-Z] R. Le Page, M. Woodroofe, and J. Zinn, Convergence to a stable distribution via order statistics, Ann. Probab. 9 (1981), 624-632.

[Lin] W. Linde, Gaussian measure of translated balls in a Banach space, Theory Probab. Appl. 34 (1989), 349-359.

[Szt] R. Sztencel, Absolute continuity of the lower tail of stable seminorms, Bull. Polish Acad. Sci. Math. 34 (1986), 231-234.

Institute of Mathematics, Technical University, Wybrzeże Wyspiańskiego 27, 50-370 Wroclaw, Poland

E-mail address: zakomath.im.pwr.wroc.pl 